\title{
Monitoring kinetic and thermodynamic parameters of fluoride adsorption from aqueous solution by pks-based anion resins
}

\author{
M.T. Bashir ${ }^{1 * 2}$, A. Salmiaton ${ }^{2}$, A. Idris ${ }^{2}$ and R. Harun ${ }^{2}$ \\ ${ }^{1}$ Department of Civil Engineering, Qurtuba University of Science and Information \\ Technology, Dera Ismail Khan, 29050, Pakistan \\ *Email: drmtb@qurtuba.edu.pk \\ Phone: +92966650228; Fax: +92966714007-8 \\ ${ }^{2}$ Department of Chemical and Environmental Engineering, Universiti Putra Malaysia, \\ 43400 UPM Serdang, Selangor, Malaysia
}

\begin{abstract}
Fluoride is important for human health, but high level of fluoride concentrations can be threatening for both humans and animals as well as damaging to the environment. This paper delineates on fluoride adsorption onto palm kernel shell-based anion resins under various fluoride concentrations and temperatures. The equilibrium fluoride adsorption capacity demonstrated by the resins was observed $2.32 \mathrm{mg} / \mathrm{g}$. Pseudo second-order model and Weber-Morris diffusion model were employed to evaluate the uptake rate and behavior of fluoride adsorption. The correlation coefficients $\left(\mathrm{R}^{2}\right)$ between experimental data and pseudo second-order model predictions was almost unity at all concentrations (5, 10 and $15 \mathrm{mg} / \mathrm{L}$ ), indicating goodness of fit of the model. On the other hand, the thermodynamic study revealed the endothermic nature of the process indicated by the positive value of enthalpy change $(2.671 \mathrm{~kJ} / \mathrm{mol})$. Moreover, the process is dominantly physical in nature along with a slight contribution of chemisorption.
\end{abstract}

Keywords: Fluoride; kinetics; thermodynamics; palm kernel shells.

\section{INTRODUCTION}

Fluoride is imperative for human health, particularly at a concentration in the range of $1.0-1.5 \mathrm{mg} / \mathrm{L}$, depending on ambient temperature. However, despite its significance, high concentrations of fluoride can be life-threatening for humans and animals and damaging to the environment [1-2]. More than 260 million populaces across the world consume drinking water with higher than recommended concentrations of fluoride [3-4]. Excessive fluoride in groundwater is a major issue because of the possibility of fluorosis arising from its bioaccumulation. Fluorosis has been identified in a number of countries including Pakistan, India, Sri Lanka, China, the United Kingdom, the United States, Argentina, South Africa, sub-Saharan Africa, and Mexico. Hence, the World Health Organization (WHO) has set the permissible limit of fluoride in drinking water to be $1.5 \mathrm{mg} / \mathrm{L}$ [5-8].

Malaysian agriculture industry including palm oil produces huge amount of numerous types of waste that is extensively used for energy production [9-10]. Remarkably, Koey et al. [11] developed a novel adsorbent for removal of dye namely RB5 by quaternization of Palm Kernel Shell (PKS). Abu-Bakar et al. [12] adopted an analogous approach for adsorption of fluoride on PKS-based adsorbent, but it could not generate exceptional results compared to study conducted by [13]. In fact, in [13] the researchers 
have changed the reaction chain as well as employing $\mathrm{KNO}_{3}$ instead of $\mathrm{NaOH}$ for mercerization process. Unlikely, they did not present the thermodynamics analysis that is essential to observe nature and spontaneity of the reaction [14] as well as exhaustive kinetic analysis by virtue of this maiden adsorbent.

In this research, palm kernel shells (PKS) based anion resins (PKS-AR) were prepared. PKS that is a waste generated in the processing of palm oil and frequently available in Malaysia, Nigeria, Indonesia and many other countries as a waste product [1011]. This study particularly provided insight into the kinetic behavior of fluoride adsorption onto PKS-AR and thermodynamics of fluoride adsorption.

\section{MATERIALS AND METHODOLOGY}

\section{Materials and Solution Preparation}

The chemicals used were potassium fluoride (KF), N-(3 Chloro-2 Hydroxypropyl) Trimethylammonium Chloride (CHMAC), and potassium hydroxide ( $\mathrm{KOH}$ pellets). All were of analytical grade (Sigma-Aldrich, Germany) and were used without further purification. A stock fluoride solution for serial dilutions was prepared by mixing $221 \mathrm{mg}$ of sodium fluoride in $1 \mathrm{~L}$ of deionized water (Milli-Q Water). Serial dilutions of the stock solution were then prepared to yield fluoride concentrations of 2.5, 5.0, 7.5, 10.0, 12.5 and $15.0 \mathrm{mg} / \mathrm{L}$. Ultimate concentrations were verified using DR-2500 Spectrophotometer (Hach) using SPADNS method.

\section{Chemical Modification of Palm Kernel Shell}

Raw PKS was cleaned and washed using hot water and treated with acetone. The PKS fibre was then dried under sunlight and ground into small particles with an effective diameter in the range of $0.25-0.5 \mathrm{~mm}$. The ground PKS was mercerized by treating it with potassium hydroxide as described in our early phase of research for fluoride adsorption [11]. In this study, the PKS was mercerized with $30 \%$ potassium hydroxide $(\mathrm{KOH})$ that facilitate the particles to swell. The mercerization process was conducted for about 2 hours at room temperature. Subsequently, the mercerized PKS was rinsed with sufficient amount of deionized water and then dried at $60{ }^{\circ} \mathrm{C}$. This process also expedites to eliminate the impurities. These mercerized particles were reacted in a mixture of $5 \mathrm{M} \mathrm{KOH}$ and CHMAC $(1: 1.5 \mathrm{v} / \mathrm{v})$ for quaternization. This process was carried out to create positive charges on the PKS-AR by grafting amine groups under alkaline conditions. Thereafter, the resins were repeatedly rinsed with a volume of deionized water (while stirring) sufficient to produce a transparent supernatant. Then the $\mathrm{pH}$ of the prepared mixture was adjusted using $1 \mathrm{M}$ sodium hydroxide and $1 \mathrm{M}$ hydrochloric acid.

\section{Fluoride Batch Study}

Batch studies were conducted at $20^{\circ} \mathrm{C}, 25{ }^{\circ} \mathrm{C} 30{ }^{\circ} \mathrm{C}, 35^{\circ} \mathrm{C}$ and $40{ }^{\circ} \mathrm{C}$ to assess adsorption data in order to ascertain maximum fluoride removal (at initial fluoride concentration: 2.5$15.0 \mathrm{mg} / \mathrm{L}$ and pH:6 ). Eq.1 is employed to observe maximum adsorbed fluoride on PKS$\mathrm{AR}$ at equilibrium.

$$
q_{e}=\frac{V\left(C_{i}-C_{e}\right)}{m}
$$

Here, $\mathrm{q}_{\mathrm{e}}$ is maximum adsorption at equilibrium $(\mathrm{mg} / \mathrm{g}), \mathrm{C}_{\mathrm{i}}$ and $\mathrm{C}_{\mathrm{e}}$ are initial, and equilibrium concentrations ( $\mathrm{mg} / \mathrm{L}$ ), respectively while $\mathrm{V}$ and $\mathrm{m}$ is the volume of adsorbate, and mass of the PKS-AR (g), respectively. On the other hand, batch studies were also 
conducted at an initial fluoride concentration of 5,10 and $15 \mathrm{mg} / \mathrm{L}$ to evaluate the kinetic parameters at temperature $30^{\circ} \mathrm{C}$.

\section{Kinetic Modeling of Nitrate Adsorption}

The kinetic behavior of fluoride removal by PKS-AR was analyzed using pseudo-second order and the Weber-Morris diffusion model. Equations (2-4) were used for calculating the requisite parameters. Moreover, Equation (2) can be modified to (3) for prediction of the initial kinetic rate in the pseudo-second order model [15].

$$
\begin{gathered}
\frac{t}{q_{t}}=\frac{1}{K_{1} q_{e}^{2}}+\frac{1}{q_{e}} t \\
\frac{t}{q_{t}}=\frac{1}{h}+\frac{1}{q_{e}} t \\
q_{t}=K_{i d} t^{0.5}+C_{i d}
\end{gathered}
$$

In (2-4), ' $t$ ' is time ( $\mathrm{min}), K_{l}$ is termed for the pseudo-second order constant (g/mg-min) and $h=\left(K_{2} \times q_{e}{ }^{2}\right)$, which defines the initial uptake rate (g/mg-min). In (4), $K_{i d}$ and $C_{i d}$ define the intra-particle diffusion constant ( $\mathrm{mg} / \mathrm{g}-\mathrm{min})$ and thickness of boundary layers, respectively.

\section{Thermodynamic Study of Fluoride Adsorption}

To evaluate the thermodynamics of adsorption, it is imperative to calculate thermodynamic parameters such as the change in free Gibbs energy ' $\Delta G^{o}$ ' $(\mathrm{kJ} / \mathrm{mol})$, entropy change ' $\Delta S^{o}$ ' $(\mathrm{kJ} / \mathrm{mol} \mathrm{K})$, and enthalpy change ' $\Delta H^{o}$ ' $(\mathrm{kJ} / \mathrm{mol})$. These parameters can be determined using Equation 5 and the Van't Hoff Equation 6.

$$
\begin{aligned}
& \Delta G^{o}=-R T \ln K \\
& \ln K=\frac{\Delta S^{o}}{R}-\frac{\Delta H^{o}}{R T}
\end{aligned}
$$

In Equation (5) and (6), $\mathrm{T}$ is absolute temperature $\left({ }^{\circ} \mathrm{K}\right)$, and $R$ is the gas constant $(8.314 \times$ $\left.10^{-3} \mathrm{~kJ} / \mathrm{mol}-\mathrm{K}\right)$. The equilibrium constant ' $K$ ' relates to the Langmuir constant ' $b$ ' measured in $(\mathrm{L} / \mathrm{mg})$ and can be obtained by multiplying ' $b$ ' by a factor of $10^{6}$ [16-17].

\section{RESULTS AND DISCUSSIONS}

\section{Effect of Initial Nitrate Concentration}

The effect of initial fluoride concentration on fluoride adsorption was monitored employing $4 \mathrm{~g} / \mathrm{L}$ of PKS-AR at a range of fluoride concentrations $(2.5-15 \mathrm{mg} / \mathrm{L})$ at temperature 20,25, 30, 35 and 40 degrees Celsius ( $\mathrm{pH}$ 6). At equilibrium, the amount of adsorbed fluoride increased from 0.52 to $2.01 \mathrm{mg} / \mathrm{g}, 0.53$ to $2.12 \mathrm{mg} / \mathrm{g}, 0.54$ to $2.21 \mathrm{mg} / \mathrm{g}$, 0.54 to $2.27 \mathrm{mg} / \mathrm{g}$ and 0.55 to $2.32 \mathrm{mg} / \mathrm{g}$ at the corresponding temperature of 20, 25, 30, 35 and 40 degrees Celsius (Figure 1).

Furthermore, the adsorption capacity of fluoride onto PKS-AR increased by an escalation in temperature that is also reported by [18] in which adsorption behavior of fluoride was studied on adsorbent using reed residues as a precursor. In addition, absorption capacities revealed that PKS-AR posses limited capacities that got exhausted as the initial fluoride concentration increased. This particular trend is analogous to work 
reported in the literature related to adsorption of fluoride and nitrate onto biomaterial based adsorbents[19-20].

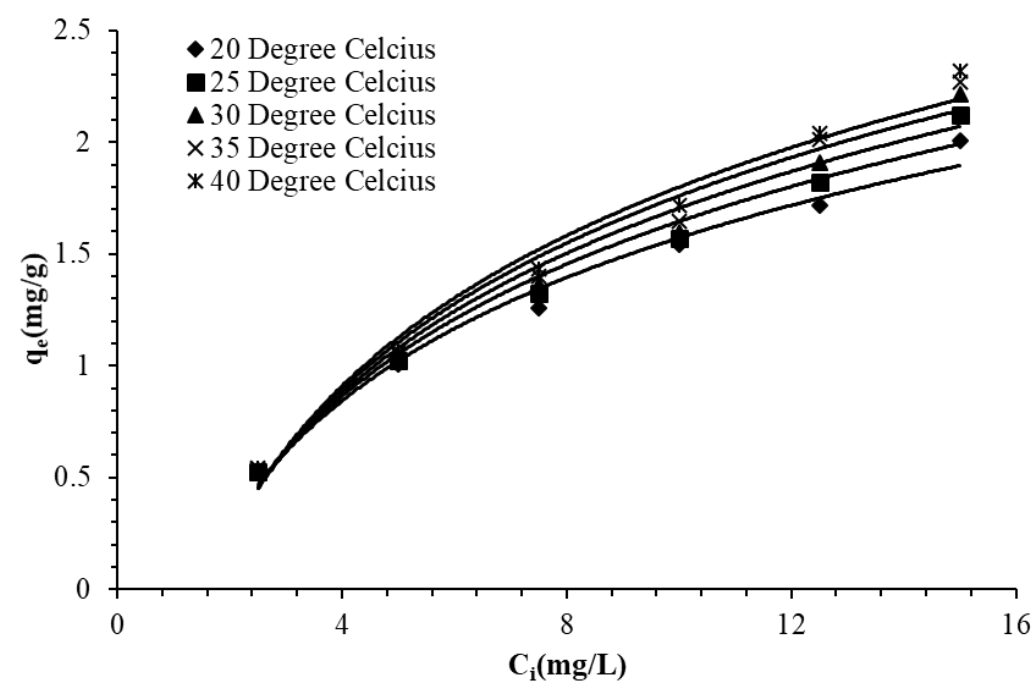

Figure 1. Fluoride adsorption capacity by PKS-AR at different temperatures (Contact time: 120 min and PKS-AR dosage: $4 \mathrm{~g} / \mathrm{L}$ ).

\section{Adsorption Kinetics}

\section{Pseudo Second-Order Study}

The constant $\mathrm{K}_{1}(\mathrm{~g} / \mathrm{mg}$-min) that represent the kinetic rate for the pseudo second-order model was calculated to be $0.2904,0.1549$, and $0.0853 \mathrm{~g} /(\mathrm{mg}$. min) at an initial fluoride concentration of 5,10 and $15 \mathrm{mg} / \mathrm{L}$, respectively. The $\mathrm{q}_{\mathrm{e}}$ value obtained for the pseudo second-order model was $1.03,1.59$, and $2.19 \mathrm{mg} / \mathrm{g}$, at different concentrations, respectively. In the absence of pseudo first-order, it is imperative to determine the value of $\mathrm{h}$ to predict initial rate constant considering modified equation (3) of pseudo second-order. The value of $\mathrm{h}$ for fluoride was found to be $1.15,2.88$ and $5.49 \mathrm{mg} / \mathrm{g}$-min describing initial rate constant of kinetics for fluoride on to PKS-AR at an initial concentration of 5, 10, and $15 \mathrm{mg} / \mathrm{L}$, respectively. These results indicate a high probability of chemisorption that largely constitutes the adsorption mechanism of fluoride onto PKS-AR [12,22].

The correlation coefficients $\left(\mathrm{R}^{2}\right)$ between experimental data and model predictions was almost unity at all concentrations under consideration (Table 1), indicating goodness of fit of the pseudosecond-order model [18]. Moreover, kinetic rate constant $\left(\mathrm{k}_{1}\right)$ decreased and initial uptake rate $(\mathrm{h})$ increased at higher concentrations of fluoride (Table 1). This phenomenon is associated to the fact that the initial concentration of fluoride was higher that facilitated adsorption but the adsorption sites on the surface of PKS-AR were blocked with the passage of time. Subsequently, adsorption rate of fluoride slowed down and gradually approached equilibrium [22-23]. 
Table 1. Summary of pseudo second-order kinetic model parameters for fluoride adsorption (pH: 6 and dose: 4g/L).

\begin{tabular}{ccccccc}
\hline Adsorbent & $\mathrm{C}_{\mathrm{i}}$ & $\mathrm{q}_{\mathrm{e}}(\mathrm{Exp})$ & \multicolumn{3}{c}{ Pseudo Second-Order Model } & $\mathrm{H}$ \\
\cline { 2 - 7 } & $(\mathrm{mg} / \mathrm{L})$ & $(\mathrm{mg} / \mathrm{g})$ & $\mathrm{K}_{1}(\mathrm{~g} / \mathrm{mg}-\mathrm{min})$ & $\mathrm{q}_{\mathrm{e}}(\mathrm{mg} / \mathrm{g})$ & $\mathrm{R}^{2}$ & $(\mathrm{mg} / \mathrm{g}-\mathrm{min})$ \\
\hline PKS-AR & 5 & 1.03 & 0.2904 & 1.07 & 0.99 & 1.15 \\
& 10 & 1.59 & 0.1549 & 1.69 & 0.99 & 2.88 \\
& 15 & 2.19 & 0.0853 & 2.34 & 0.99 & 5.49 \\
\hline
\end{tabular}

\section{Intra-particle Diffusion}

The external mass transfer that can also be referred as boundary diffusion as well as describe rate limiting steps which are imperative regarding the solute-transfer process in solid-liquid adsorption [18]. The rate-limiting step in the adsorption of fluoride by PKSAR was investigated by plotting (Figure 2) the amount of fluoride adsorbed against the square root of time ( at all initial concentrations of fluoride under consideration ), based on the Weber and Morris model (Equation 4). Figure 2 highlights the variable rate of fluoride adsorption on PKS-AR, with an initial phase influenced by a boundary layer diffusion effect, a second phase controlled by intra-particle diffusion, and a final phase displayed an equilibrium stage. Thus, Figure 2 specifies that the process of fluoride removal on PKSAR was rather complex and influenced by both surface adsorption and the intra-particle diffusion [12,19]. Moreover, summarization of intra-particle parameters for fluoride uptake on to PKS-AR is presented in Table 2. These results demonstrate that intra-particle diffusion increased with corresponding increase in fluoride concentration in the solution that is analogous to research reported in $[12,18]$.

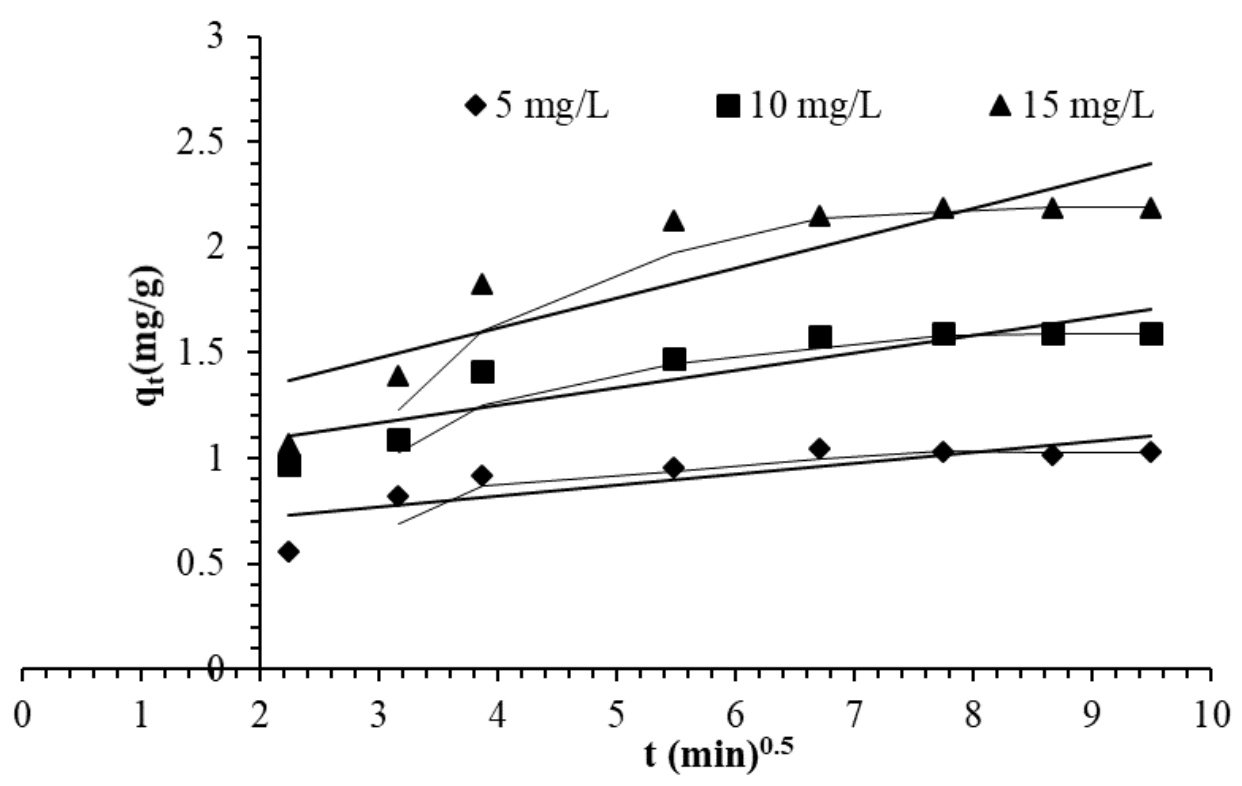

Figure 2. Amount of fluoride adsorbed versus the square root of time, highlighting the effect of intra-particle diffusion (PKS-AR dosage: 4g/L, $\mathrm{pH}: 6$ ). 
Table 2. Summarization of intra-particle parameters for fluoride uptake on to PKS-AR.

\begin{tabular}{lcccc}
\hline Specie & $\begin{array}{c}\mathrm{C}_{\mathrm{i}} \\
(\mathrm{mg} / \mathrm{L})\end{array}$ & $\begin{array}{c}\mathrm{K}_{\mathrm{id}} \\
(\mathrm{mg} / \mathrm{g}-\mathrm{min})\end{array}$ & $\mathrm{C}_{\mathrm{id}}$ & $\mathrm{R}^{2}$ \\
\cline { 2 - 5 } Fluoride & 5 & $4.21 \times 10^{-2}$ & 0.617 & 0.688 \\
& 10 & $12.80 \times 10^{-2}$ & 0.471 & 0.670 \\
& 15 & $11.63 \times 10^{-2}$ & 1.047 & 0.762 \\
\hline
\end{tabular}

\section{Thermodynamics of Fluoride Adsorption}

Thermodynamic parameters for fluoride were studied at a temperature of 293, 298, 303, 308 and $313 \mathrm{~K}\left(20,25,30,35\right.$ and $40{ }^{\circ} \mathrm{C}$. Langmuir plots for fluoride adsorption on PKSAR are shown in Figure 3, and the Van't Hoff (6) is used to plot $\ln K$ versus $1 / T$ that provided a value of 0.905 for $\mathrm{R}^{2}$ (Figure 4). The values of $\Delta G^{o}, \Delta H^{o}$, and $\Delta S^{o}$ were determined by (5) and (6) as well as from plot of $\operatorname{lnK}$ versus $1 / \mathrm{T}$. The values of $\Delta G^{o}$ for fluoride were calculated as $-33.15,-33.74,-34.34,34.94$ and -35.61 for corresponding temperature values of 293, 298, 303, 313 and $313 \mathrm{k}$ (Tables 3). The standard Gibbs free energy has a negative value, and this basically describes the spontaneity of the fluoride sorption process at all the temperature values observed in the process [12,19]. Moreover, the nature of the interaction between fluoride onto PKS-AR was found to be endothermic indicated by the positive value of ' $\Delta H^{o}$ ' for respective contaminants (Table 3 ). The values of the $\Delta H^{\circ}$ were observed to be $2.671 \mathrm{~kJ} / \mathrm{mol}$ which is lower than $40 \mathrm{~kJ} / \mathrm{mol}$ indicating the adsorption process dominantly physical in nature [24] despite significant contribution chemisorption that is apparent from the goodness of fit of pseudo-second-order.

Furthermore, the adsorption of fluoride showed more affinity at higher temperatures because of endothermic nature of the process. This finding is analogous as described by [3] in fluoride adsorption on adsorbent prepared by the fungus (P.Elrengi). Also, the change in standard entropy for adsorption process is positive (Table 3 ) showing increasing degree of freedom of fluoride ions. This phenomenon is associated to change in the structure of PKS-AR that increased the solid-liquid interface $[3,18,20]$.

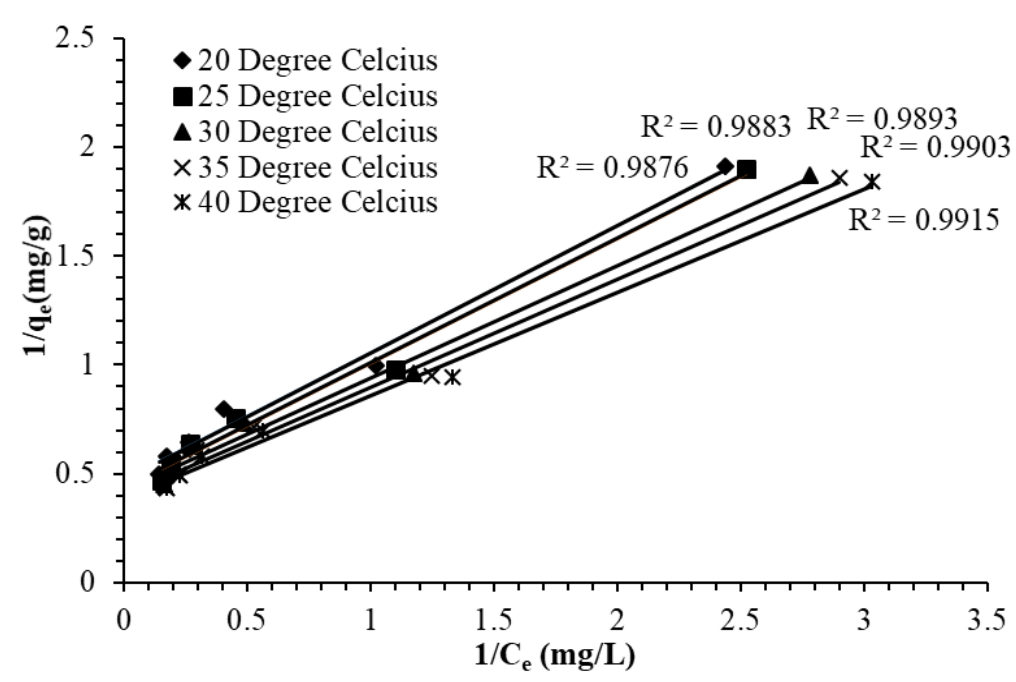

Figure 3. Langmuir plots for fluoride adsorption on PKS-AR at various temperatures (PKS-AR Dosage: 4g/L). 


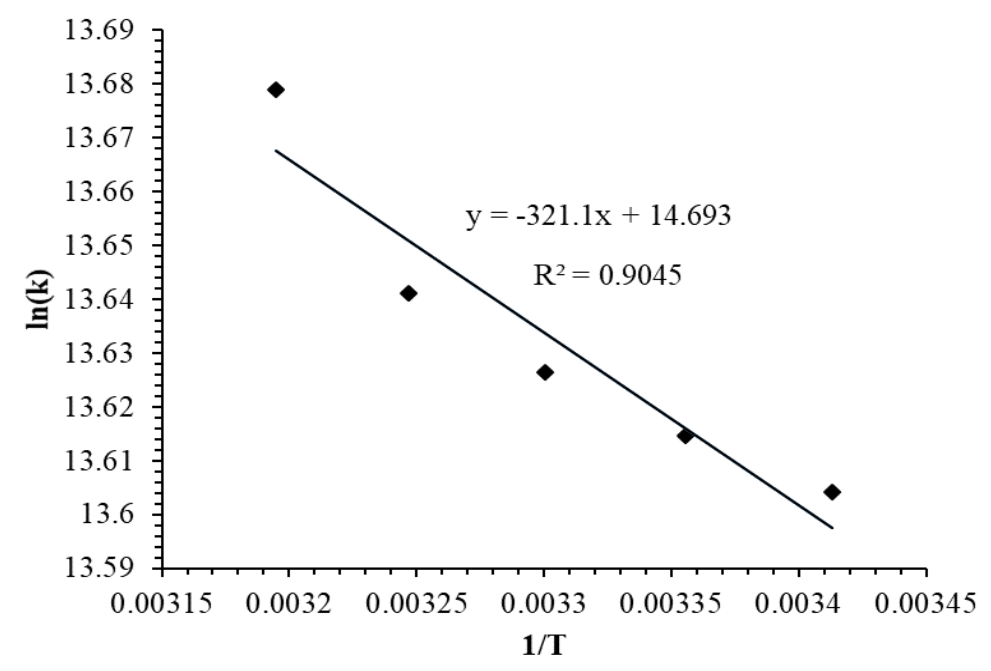

Figure 3. Presentation of Van't Hoff plot of $1 / \mathrm{T}$ versus $\ln (\mathrm{K})$ for fluoride adsorption on PKS-AR.

Table 3. Thermodynamics parameters for adsorption of fluoride on PKS-AR.

\begin{tabular}{lccccc}
\hline $\begin{array}{l}\text { Temp. } \\
(\mathrm{K})\end{array}$ & $\begin{array}{c}\mathrm{b} \\
(\mathrm{L} / \mathrm{mg})\end{array}$ & $\mathrm{K}$ & $\begin{array}{c}\Delta \mathrm{G}^{\mathrm{o}} \\
(\mathrm{kJ} / \mathrm{mol})\end{array}$ & $\begin{array}{c}\Delta \mathrm{H}^{\mathrm{o}} \\
(\mathrm{kJ} / \mathrm{mol})\end{array}$ & $\begin{array}{c}\Delta \mathrm{S}^{\mathrm{o}} \\
(\mathrm{kJ} /(\mathrm{mol}-\mathrm{K})\end{array}$ \\
\hline 293 & 0.809507 & 809507 & -33.15 & & \\
298 & 0.818019 & 818019 & -33.74 & & \\
303 & 0.827684 & 827684 & -34.34 & 2.671 & 0.122 \\
308 & 0.840014 & 840014 & -34.94 & & \\
313 & 0.872342 & 872342 & -35.61 & & \\
\hline
\end{tabular}

\section{CONCLUSION}

The novel adsorbent (PKS-AR) prepared by chemical modification of palm kernel shells effectively adsorbed $2.32 \mathrm{mg} / \mathrm{g}$ of fluoride from water at $\mathrm{pH}$. The correlation coefficients $\left(\mathrm{R}^{2}\right)$ between experimental data and Pseudo-second order prediction was almost unity at all concentrations. On the other hand, intra-particle diffusion model reveals variable rate of fluoride adsorption on PKS-AR showing boundary layer diffusion followed by intraparticle diffusion before approaching an equilibrium stage in the final phase. Moreover, at an initial fluoride concentration of 5,10 and $15 \mathrm{mg} / \mathrm{L}$, initial uptake rate of fluoride is very high that is observed $1.15,2.88$ and $5.49 \mathrm{mg} / \mathrm{g}$-min, respectively. Thermodynamics of adsorption for fluoride revealed that the nature of the interaction between fluoride onto PKS-AR was endothermic indicated by the positive value of ' $\Delta H o$ ' that was $2.671 \mathrm{~kJ} / \mathrm{mole}$. Furthermore, the adsorption of fluoride showed more affinity at higher temperatures and chemisorption is also a vital mechanism in the fluoride adsorption process along with the dominance of physical adsorption. 


\section{ACKNOWLEDGEMENT}

The authors would like to acknowledge the Qurtuba University of Science and IT, DI Khan, Pakistan and Universiti Putra Malaysia, Serdang, Malaysia for the provision of financial support. All the equipment and development of adsorbent was done using the property of the Department of Chemical and Environmental Engineering, UPM, Malaysia.

\section{REFERENCES}

[1] Mohan D, Kumar S, Srivastava A. Fluoride removal from ground water using magnetic and nonmagnetic corn stover biochars. Ecological Engineering. 2014; 73:798-808.

[2] Avvannavar S, Fawell J, Bailey J. Chilton E, Dahi L, Fewtrell L, Magara Y. Fluoride in drinking-water, 1st ed. 2007; London: WHO, IWA Publishers.

[3] Amin F, Talpur FN, Balouch A, Surhio MA, Bhutto MA. Biosorption of fluoride from aqueous solution by white-rot fungus pleurotus eryngii ATCC 90888, Environmental Nanotechnology, Monitoring and Management. 2015; 3:30-37.

[4] UNICEF, UNICEF - Official Position on Water Fluoridation. Retrieved from http://www.nofluoride.com/unicef_fluor.htm dated on 10 May 2017.

[5] Gautam N, Singh R. Removal of fluoride from ground water by thermally activated neem (Azadiractica Indica) and peepal (Ficus Religiosa) leaves carbon adsorbents. World Journal of Pharmacy and Pharmaceutical Sciences. 2017; 6:1050-1057.

[6] Bello SO, Adegoke AK, Akinyunni O. Preparation and characterization of a novel adsorbent from Moringa oleifera leaf. Applied Water Science. 2015; 7:1295-1305.

[7] Rout T, Verma R, Dennis R, Banerjee S. Study the removal of fluoride from aqueous medium by using nano-composites. Journal of Encapsulation and Adsorption Sciences. 2015; 5:38-52.

[8] Mukhopadhyay K, Ghosh A, Das S, Show B, Sasikumar P, Ghosh, CU. Synthesis and characterisation of cerium(iv)-incorporated hydrous iron(iii) oxide as an adsorbent for fluoride removal from water, RSC Advances. 2017; 7: 26037-51.

[9] Al-Kayiem HH, Yunus YM. Drying of empty fruit bunches as wasted biomass by hybrid solar-thermal drying technique. Journal of Mechanical Engineering and Sciences. 2013; 5:652-661.

[10] Ibrahim MS, Sapuan SM, Faieza AA. Mechanical and thermal properties of composites from unsaturated polyester filled with oil palm ash. Journal of Mechanical Engineering and Sciences. 2012; 2:133-147.

[11] Koay Y, Ahamad I, Nourouzi MM, Abdullah L, Choong T. Development of novel low-cost quaternized adsorbent from palm oil agriculture waste for reactive dye removal. BioResources. 2014; 9: 66-85.

[12] Abu-Bakar A, Koay Y, Ching Y, Abdullah L, Choong T, Alkhatib M, Mobarekeh M, Zahri MN. Removal of fluoride using quaternized palm kernel shell as adsorbents: equilibrium isotherms and kinetics studies. BioResources. 2016; 11:4485-511.

[13] Bashir MT, Salmiaton A, Idris A, Harun R, Nourouzi MM. Fluoride removal by chemical modification of palm kernel shell-based adsorbent: A novel agricultural waste utilization approach. Asian Journal of Microbiology, Biotechnology \& Environmental Sciences. 2015; 17:533-42.

[14] Xu L, Zheng X, Cui H, Zhu Z, Liang J, Zhou J. Equilibrium, kinetic, and thermodynamic studies on the adsorption of cadmium from aqueous solution by modified biomass ash. Bioinorganic Chemistry and Applications. 2017; 2017:1-9. 
[15] Ho Y, McKay G. Pseudo-second order model for sorption processes. Process Biochemistry. 1999; 34:451-65.

[16] Bashir MT, Salmiaton A, Idris A, Harun R. Kinetic and thermodynamic study of nitrate adsorption from aqueous solution by lignocellulose-based anion resins, Desalination and Water Treatment. 2017; 62: 449-56.

[17] Keränen A, Leiviskä T, Hormi O, Tanskanen J. Removal of nitrate by modified pine sawdust: Effects of temperature and co-existing anions. Journal of Environmental Management. 2015; 147:46-54.

[18] Song R, Yang S, Xu H, Wang Z, Chen Y, Wang Y. Adsorption behavior and mechanism for the uptake of fluoride ions by reed residues. International Journal of Environmental Research and Public Health.2018;15:101-112.

[19] Yadav A, Abbassi R, Gupta A, Dadashzadeh M. Removal of fluoride from aqueous solution and groundwater by wheat straw, sawdust and activated bagasse carbon of sugarcane, Ecological Engineering. 2013;52: 211-218.

[20] Xu X, Gao B, Tan X, Yue Q, Zhong Q. Li Q. Characteristics of amine-crosslinked wheat straw and its adsorption mechanisms for phosphate and chromium (VI) removal from aqueous solution. Carbohydrate Polymers. 2011; 84: 1054-60.

[21] Katal R, Baei M, Rahmati H, Esfandian H.Kinetic, isotherm and thermodynamic study of nitrate adsorption from aqueous solution using modified rice husk. Journal of Industrial and Engineering Chemistry. 2012; 18:295-302.

[22] Abri A, Tajbakhsh M, Sadeghi A. Adsorption of fluoride on a chitosan-based magnetic nanocomposite: equilibrium and kinetics studies. Water Science and Technology: Water Supply.2018; 18: ws2018050.

[23] Iriel A, Bruneel S, Schenone N, Cirelli A. The removal of fluoride from aqueous solution by a lateritic soil adsorption: Kinetic and equilibrium studies. Ecotoxicology and Environmental Safety. 2018; 149:166-172.

[24] Bhatnagar A, Ji M, Choi Y, Jung W, Lee S, Kim, G. Lee G, Suk H, Kim H, Min B, Kim S, Jeon B, Kang J. Removal of nitrate from water by adsorption onto zinc chloride treated activated carbon. Separation Science and Technology. 2008; 43: 886-907. 